\title{
The Classical and Quantum Inflaton: the Precise Inflationary Potential and Quantum Inflaton Decay after WMAP
}

\author{
D. Boyanovsky ${ }^{a} \mathrm{~b} *, \underline{\text { H. J. de Vega }}{ }^{\mathrm{b}} \mathrm{a}^{\dagger}$, N. G. Sanchez ${ }^{\mathrm{b}}$.

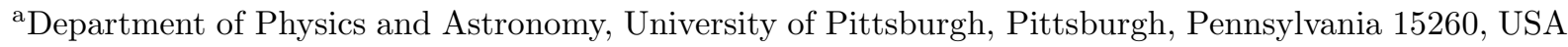 \\ ${ }^{\mathrm{b}}$ Observatoire de Paris, LERMA. Laboratoire Associé au CNRS UMR 8112. \\ 61, Avenue de l'Observatoire, 75014 Paris, France. \\ ${ }^{c}$ LPTHE, Université Pierre et Marie Curie (Paris VI) et Denis Diderot (Paris VII), Laboratoire Associé \\ au CNRS UMR 7589, Tour 24, 5ème. étage, 4, Place Jussieu, 75252 Paris, Cedex 05, France.
}

We clarify classical inflaton models by considering them as effective field theories à la Ginzburg-Landau. In this approach, the WMAP statement excluding the pure $\phi^{4}$ potential implies the presence of an inflaton mass term at the scale $m \sim 10^{13} \mathrm{GeV}$. Chaotic, new and hybrid inflation models are studied in an unified manner. In all cases the inflaton potential takes the form $V(\phi)=m^{2} M_{P l}^{2} v\left(\frac{\phi}{M_{P l}}\right)$, where all coefficients in the polynomial $v(\varphi)$ are of order $\left(m / M_{P l}\right)^{0}$. If such potential corresponds to supersymmetry breaking, the corresponding susy breaking scale is $\sqrt{m M_{P l}} \sim 10^{16} \mathrm{GeV}$ which turns out to coincide with the grand unification (GUT) scale. The inflaton mass is therefore given by a see-saw formula $m \sim M_{G U T}^{2} / M_{P l}$. For red tilted spectrum, the potential which fits the best the present data $\left(\left|1-n_{s}\right| \lesssim 0.1, r \lesssim 0.1\right)$ and which best prepares the way for the forthcoming data is a trinomial polynomial with negative quadratic term (new inflation). For blue tilted spectrum, hybrid inflation turns to be the best choice. In both cases, we find an analytic formula relating the inflaton mass with the ratio $r$ of tensor to scalar perturbations and the spectral index $n_{s}$ of scalar perturbations: $10^{6} \frac{m}{M_{P l}}=127 \sqrt{r\left|1-n_{s}\right|}$ where the numerical coefficient is fixed by the WMAP amplitude of adiabatic perturbations. Implications for string theory are discussed. We then review quantum phenomena during inflation which contribute to relevant observables in the CMB anisotropies and polarization and we focus on inflaton decay. The deviation from the scale invariant power spectrum measured by a small parameter $\Delta$ turns to be crucial, $\Delta$ regulates the infrared too. In slow roll inflation, $\Delta$ is a simple function of the slow roll parameters. We find that quantum fluctuations can self-decay as a consequence of the inflationary expansion through processes which are forbidden in Minkowski space-time. We compute the self-decay of the inflaton quantum fluctuations during slow roll inflation: for wavelengths deep inside the Hubble radius the decay is enhanced by the emission of ultrasoft collinear quanta, i.e. bremsstrahlung radiation of superhorizon quanta which becomes the leading decay channel for physical wavelengths $H \ll k_{p h}(\eta) \ll H /\left(\eta_{V}-\epsilon_{V}\right)$. The decay of short wavelength fluctuations hastens as the physical wave vector approaches the horizon. Superhorizon fluctuations decay with a power law $\eta^{\Gamma}$ in conformal time where $\Gamma$ is expressed in terms of the amplitude of curvature perturbations $\triangle_{\mathcal{R}}^{2}$, the scalar spectral index $n_{s}$, the tensor to scalar ratio $r$ and slow roll parameters. The behavior of the growing mode $\eta^{\eta_{V}-\epsilon_{V}+\Gamma} / \eta$ features a new scaling dimension $\Gamma$. We discuss the implications of these results for scalar and tensor perturbations as well as for non-gaussianities in the power spectrum. The recent WMAP data suggests $\Gamma \gtrsim 3.6 \times 10^{-9}$.

\footnotetext{
*boyan@pitt.edu

$\dagger$ devega@lpthe.jussieu.fr

‡Norma.Sanchez@obspm.fr
}

\section{Introduction}

A period of accelerated expansion in the early universe, namely inflation, is nowadays part of standard cosmology since explains the homogeneity, isotropy and flatness of the observed Uni- 
verse 11. At the same time, inflation provides a mechanism for generating metric fluctuations which seed large scale structure: during inflation physical scales grow faster than the Hubble radius but slower than it during both radiation or matter domination eras, therefore physical wavelengths cross the horizon (Hubble radius) twice. Quantum fluctuations generated during inflation with wavelengths smaller than the Hubble radius become classical and are amplified upon first crossing the horizon. As they re-enter the horizon during the decelerated stage these fluctuations provide the seed for matter and radiation inhomogeneities which generate structure upon gravitational collapse. Most of the inflationary models predict fairly generic features: a gaussian, nearly scale invariant spectrum of adiabatic scalar and tensor primordial perturbations (gravitational waves). These generic predictions are in spectacular agreement with Cosmic Microwave Background (CMB) observations. Gaussian [5] and adiabatic nearly scale invariant primordial fluctuations 6 provide an excellent fit to the WMAP data as well as to a variety of large scale structure observations. Perhaps the most striking confirmation of inflation as the mechanism for generating superhorizon ('acausal') perturbations is the anticorrelation peak in the temperaturepolarization (TE) angular power spectra at $l \sim$ 150 corresponding to superhorizon scales [78]. The anticorrelation between the E-mode (parity even) polarization fluctuation and the temperature fluctuation is a distinctive feature of superhorizon adiabatic fluctuations 9]: the (peculiar) velocity gradient generates a quadrupole temperature anisotropy field around electrons which in turn generates an E-polarization mode. By continuity, the gradient of the peculiar velocity field is related to the time derivative of the density (temperature) fluctuations, hence the peculiar velocity and the initial (adiabatic) contribution to the (acoustic) oscillations of the photon baryon fluid are out of phase by $\pi / 2$. . Thus, the product of these two terms gives an anticorrelation peak at $k c_{s} \eta_{\text {dec }}=3 \pi / 4$ which corresponds to superhorizon wavelengths since the size of the horizon is $\sqrt{3}$ larger than the size of the sound horizon. The WMAP (TE) data 78 clearly displays this
(TE) anticorrelation peak at $l \sim 150$ providing perhaps one of the most striking confirmations of adiabatic superhorizon fluctuations as predicted by inflation. While the robust predictions of a generic inflationary model provide an excellent fit to the WMAP data, it is still necessary to decide between a host of possible models $[3$. In addition, potential experimental deviations from the most generic features are the focus of intense study as the low angular momentum depletion. With the ever increasing precision of CMB measurements, it is conceivable that forthcoming observations will allow a substantial progress in singling out inflationary models [3]. Relevant discriminants between models are: non-gaussianity, deviations from constant scaling exponents (running spectral index) either for scalar and/or tensor perturbations, an isocurvature component of primordial fluctuations, etc. Quantum effects associated with interactions can potentially lead to non-gaussian correlations [2, [10- 15]. Therefore the detection of deviations from constant scaling exponents (as hinted in the WMAP data) or small non-gaussianities in the temperature correlations imply potentially interesting quantum phenomena during the inflationary stage that were imprinted on superhorizon scales.

The inflaton is usually studied as a homogeneous classical scalar field [1]. However, important aspects of the dynamics require a full quantum treatment for their consistent description. The quantum dynamics of the inflaton is systematically treated within a non-perturbative framework and consequences on the CMB anisotropy spectrum were analyzed in ref. [16].

\section{Classical Inflation as an Effective Field Theory: the Best Inflationary Potential from the WMAP Data}

The classical dynamics of the inflaton (a massive scalar field) coupled to a cosmological background clearly shows that inflationary behaviour is an attractor 4 4 . This is a generic and robust feature of inflation. The robust predictions of inflation (value of the entropy of the universe, solution of the flatness problem, a small amplitude almost scale invariant power spectrum of adia- 
batic Gaussian density fluctuations explaining the CMB anisotropies) which are common to many available inflationary scenarios, show the predictive power of the inflationary paradigm. Whatever the microscopic model for the early universe (GUT theory, string theory) would be, it should include inflation with the generic features we know today.

Inflationary dynamics is typically studied by treating the inflaton as a homogeneous classical scalar field 1 whose evolution is determined by a classical equation of motion, while the inflaton quantum fluctuations (around the classical value and in the Gaussian approximation) provide the seeds for the scalar density perturbations of the metric. In quantum field theory, this classical inflaton corresponds to the expectation value of a quantum field operator in a translational invariant state. Important aspects of the inflationary dynamics, as resonant particle production and the nonlinear back-reaction that it generates, require a full quantum treatment of the inflaton for their consistent description. The quantum dynamics of the inflaton in a non-perturbative framework and its consequences on the CMB anisotropy spectrum were treated in refs. 16. Particle decay in a de Sitter background has been studied in refs. [1718] and during slow roll inflation in ref. [2] together with its implication for the decay of the density fluctuations.

Inflation as known today should be considered as an effective theory, that is, it is not a fundamental theory but a theory of a condensate (the inflaton field) which follows from a more fundamental one (the GUT model, string theory). The inflaton field $\phi$ may not correspond to any real particle (even unstable) but is just an effective description while the microscopic description should come from the GUT model. At present, there is no derivation of the inflaton model from more microscopic theories, either GUT models or string theories. However, the relation of inflation to these microscopic theories is akin to the relation between the effective Ginzburg-Landau theory of superconductivity and the microscopic BCS theory, or the relation between the $O(4)$ sigma model of low lying mesons and quantum chromodynamics (QCD).
We provide in ref. 3 a clear understanding of inflation and the inflaton potential from effective field theory and the WMAP data. This clearly places inflation within the perspective and understanding of effective theories in particle physics. In addition, it sets up a clean way to directly confront the inflationary predictions with the forthcoming CMB data and select a definitive model.

The following inflaton potential or alternatively the hybrid inflation model are rich enough to describe the physics of inflation and accurately reproduce the available data 5678 :

$V(\phi)=\left|m^{2}\right| M_{P l}^{2}\left[v_{0} \pm \frac{1}{2} \varphi^{2}+\frac{2}{3} \gamma \varphi^{3}+\frac{1}{32} \kappa \varphi^{4}\right]$.

Here $\varphi \equiv \frac{\phi}{M_{P l}},|m| \sim 10^{13} \mathrm{GeV}$, the dimensionless parameters $\gamma$ and $\kappa$ are of order $\left(\frac{m}{M_{P l}}\right)^{0}$, and $v_{0}$ is such that $V(\phi)$ and $V^{\prime}(\phi)$ vanish at the absolute minimum of $V(\phi)$. This ensures that inflation ends after a finite time with a finite number of efolds. $\kappa$ must be positive to ensure stability while $\gamma$ and the mass term $\varphi^{2}$ can have either sign. $\gamma$ describes how asymmetric is the potential while $\kappa$ determines how steep it is. After factoring out the scales $m$ and $M_{P}$ there remains the small quantity $1 / N_{\text {efolds }} \sim \mathcal{O}\left(10^{-2}\right)$ which determines the departure from scale invariance as well as the scalar to tensor ratio in this effective description $\left[3\right.$. ( $N_{\text {efolds }}$ being the number of efolds from the first horizon crossing to the end of inflation).

The potential eq. (1) cover a wide class of inflationary scenarios: small field scenarios (new inflation) for spontaneously broken symmetric potentials (negative mass square), as well as large field scenarios (chaotic inflation) for unbroken symmetric potentials (positive mass square). Coupling the inflaton to another scalar field yields the hybrid type scenarios. Renormalizability restricts the degree of the potential eq.(1) to four. Indeed, in the context of effective theories potentials of any degree may be considered but a quartic potential is rich enough to describe the full physics and to reproduce accurately the WMAP data.

In the context of an effective theory or 
Ginzburg-Landau model it is highly unnatural to drop the quadratic term $\varphi^{2}$. This is to exactly choose the critical point of the model $m^{2}=0$. In fact, the recent WMAP 5678 statement disfavouring the monomial $\varphi^{4}$ potential precisely supports a generic polynomial inflaton potential as in eq.(11). Excluding the quadratic mass term in the potential $V(\phi)$ implies to fine tune the mass to zero which is only justified at isolated (critical) points. Therefore the pure quartic potential $\varphi^{4}$ is physically an unnatural choice implying fine tuning to zero the coefficient of $\varphi^{2}$.

We obtain analytic and unifying expressions for chaotic and new inflation for the relevant observables 3: the amplitude for scalar fluctuations $\left|\delta_{k_{\text {add }}}^{(S)}\right|^{2}$, spectral index $n_{s}$ and ratio $r$ of tensor to scalar perturbations as well as for hybrid inflation and plot them for the three scenarios. Particularly interesting are the plots of $n_{s}$ vs. $r$ [3].

We express the ratio of the inflaton mass and the Planck mass $x \equiv 10^{6} \frac{m}{M_{P l}}$ in terms of the amplitude of adiabatic perturbations and the parameters in the potential. Furthermore, we can express $x$ in terms of observable quantities as $r$ and $n_{s}$. We find for new inflation when both $r$ and $\left|n_{s}-1\right|$ are small,

$$
\begin{array}{r}
10^{6} \frac{m}{M_{P l}}=x=5 \pi \sqrt{3} 10^{5}\left|\delta_{k a d}^{(S)}\right| \sqrt{r\left(1-n_{s}\right)}= \\
=127 \sqrt{r\left(1-n_{s}\right)} \pm 6 \%,
\end{array}
$$

where the $\pm 6 \%$ correspond to the error bars in the amplitude of adiabatic perturbations 5678 . We analyze in ref. 3] how the mass ratio $\frac{m}{M_{P l}}$ varies with $n_{s}$ and $r$. We find a limiting value $x_{0} \equiv 10^{5} \frac{m_{0}}{M_{P l}} \simeq 1$ for the inflaton mass such that $m_{0} \simeq 10^{-5} M_{P l}$ is a minimal inflaton mass for chaotic inflation, and a maximal mass for new inflation in order to keep $n_{s}$ and $r$ within the WMAP data.

New inflation arises for broken symmetric potentials (the minus sign in front of the $\varphi^{2}$ term) while chaotic inflation appears both for unbroken and broken symmetric potentials. For broken symmetry, we find that analytic continuation connects the observables for chaotic and new inflation: the observables are two-valued functions of $y \equiv \kappa N_{\text {efolds }}$. One branch corresponds to new inflation and the other branch to chaotic inflation. $n_{s}, r$ and $\left|\delta_{k \text { ad }}^{(S)}\right|^{2}$ for chaotic inflation are connected by analytic continuation to the same quantities for new inflation. The branch point where the two scenarios connect corresponds to the monomial $+\frac{1}{2} \varphi^{2}$ potential $(\kappa=\gamma=0)$.

The potential which best fits the present data for a red tilted spectrum $\left(n_{s}<1\right)$ and which best prepares the way to the expected data (a small $r \lesssim 0.1)$ is given by the trinomial potential eq.(11) with a negative $\varphi^{2}$ term, that is new inflation.

In new inflation we have the upper bound

$$
r \leq \frac{8}{N_{\text {efolds }}} \simeq 0.16 \quad .
$$

This upper bound is attained by the quadratic monomial potential. On the contrary, in chaotic inflation for both signs of the $\varphi^{2}$ term, $r$ is bounded as

$$
0.16 \simeq \frac{8}{N_{\text {efolds }}}<r<\frac{16}{N_{\text {efolds }}} \simeq 0.32,
$$

This bound holds for all values of the cubic coupling $\gamma$ which describes the asymmetry of the potential. The lower and upper bounds for $r$ are saturated by the quadratic and quartic monomials, respectively.

If an upper bound $r \leq 0.16$ turns out to be measured eq.(3) implies that chaotic inflation is excluded.

For chaotic and new inflation, we find the following properties $[$ ] :

- $n_{s}$ is bounded as

$$
\begin{aligned}
& n_{s} \leq 1-\frac{2}{N_{\text {efolds }}} \simeq 0.96 \quad \text { chaotic inflation, } \\
& n_{s} \leq 1-\frac{1.558005 \ldots}{N_{\text {efolds }}} \simeq 0.9688 \quad \text { new inflation } .
\end{aligned}
$$

The value at the bound for chaotic inflation corresponds to the quadratic monomial potential.

- $n_{s}$ decreases with the steepness $\kappa$ for fixed asymmetry $h \equiv \gamma \sqrt{\frac{8}{\kappa}}<0$ and $n_{s}$ grows with the asymmetry $|h|$ for fixed steepness $\kappa$. 
For the general trinomial potential eq.(1), $r$ decreases with $n_{s}$ in chaotic inflation while, in new inflation, $r$ grows with $n_{s}$. The trinomial potential eq. (11) can yield very small $r$ for red tilt with $n_{s}<1$ and near unit for new inflation.

Hybrid inflation always gives a blue tilted spectrum $n_{s}>1$ in the $\Lambda$-dominated regime, allowing $n_{s}-1$ and $r$ to be small. Interestingly enough, we obtain for hybrid inflation a formula for the mass ratio $x$ with a similar structure to eq.(2) for new inflation:

$$
x=10^{6} \frac{m}{M_{P l}}=127 \sqrt{r\left(n_{s}-1+\frac{3}{8} r\right)} .
$$

As shown in ref. [3], $\frac{m}{M_{P l}}$ decreases when $r$ and $n_{s}-1$ both approach zero. We relate the cosmological constant in the hybrid inflation Lagrangian with the ratio $r$ as

$$
\frac{\Lambda_{0}}{M_{P l}^{4}}=0.329 \times 10^{-7} r
$$

and we find that $\left(n_{s}-1\right)$ gives an upper bound on the cosmological constant:

$$
\frac{\Lambda_{0}}{m^{2} M_{P l}^{2}}<\frac{2}{n_{s}-1} .
$$

In summary, for small $r \lesssim 0.1$ and $n_{s}$ near unit, new inflation from the trinomial potential eq.(1) and hybrid inflation emerge as the best inflation candidates. Whether $n_{s}$ turns to be larger or smaller than one will choose hybrid inflation or new inflation, respectively. In any case $\left|n_{s}-1\right|$ turns to be of order $1 / N_{\text {efolds }}$. This can be understood intuitively as follows: the geometry of the universe is scale invariant during de Sitter stage since the metric takes in conformal time the form

$$
d s^{2}=\frac{1}{(H \eta)^{2}}\left[(d \eta)^{2}-(d \vec{x})^{2}\right] .
$$

Therefore, the primordial power generated is scale invariant except for the fact that inflation is not eternal and lasts for $N_{\text {efolds. }}$. Hence, the primordial spectrum is scale invariant up to $1 / N_{\text {efolds }}$ corrections. Also, the ratio $r$ turns to be of order $1 / N_{\text {efolds }}$ (chaotic and new inflation) or $1 / N_{\text {efolds }}^{2}$ (hybrid inflation).
Generally speaking, the amplitude of adiabatic perturbations is given in order of magnitude by 3

$$
\left|\delta_{k \text { ad }}^{(S)}\right| \sim N_{\text {efolds }} \frac{|m|}{M_{P l}} .
$$

\section{Implications for Supersymmetry and String Theory}

In order to reproduce the CMB data, the inflationary potentials in the slow roll scenarios must have the structure

$$
V(\phi)=M^{4} v\left(\frac{\phi}{M_{P l}}\right)
$$

where $v(0)=v^{\prime}(0)=0$ and all higher derivatives at the origin are of the order one. The inflaton mass is therefore given by a see-saw-like formula

$$
m \simeq \frac{M^{2}}{M_{P l}}
$$

As stated above, the WMAP data imply $m \sim$ $10^{13} \mathrm{GeV}$, Eq. (5) implies that $M$ is precisely at the grand unification scale $M \sim 10^{16} \mathrm{GeV}[1$.

Grand unification proposes that at some energy scale all three couplings (electromagnetic, weak and strong) should merge into one. In this case, such grand unified scale turns out to be $E \sim 10^{16} \mathrm{GeV}[2325$.

Three strong independent indications of this scale are available nowadays: 1) the convergence of the running electromagnetic, weak and strong couplings, 2) the large mass scale to explain the neutrino masses via the see-saw mechanism and 3 ) the scale $M$ in the above inflaton potential. Also, notice that eq. (5) has the structure of the moduli potential coming from supersymmetry breaking. Therefore, the supersymmetry breaking scale would be at the GUT scale too.

The running of the couplings with the energy (or the length) is governed by the renormalization group. For the standard model of electromagnetic, weak and strong interactions, the renormalization group yields that the three couplings get unified approximately at $\sim 10^{16} \mathrm{GeV}$. A better convergence is obtained in supersymmetric extensions of the standard model 2325$]$. 
Neutrino oscillations and neutrino masses are currently explained in the see-saw mechanism as follows 26],

$$
\Delta m_{\nu} \sim \frac{M_{F e r m i}^{2}}{M}
$$

where $M_{\text {Fermi }} \sim 250 \mathrm{GeV}$ is the Fermi mass scale, $M \gg M_{\text {Fermi }}$ is a large energy scale and $\Delta m_{\nu}$ is the difference between the neutrino masses for different flavors. The observed values for $\Delta m_{\nu} \sim 0.009-0.05 \mathrm{eV}$ naturally call for a mass scale $M \sim 10^{15-16} \mathrm{GeV}$ close to the GUT scale 26 .

Eq.(4) for the inflaton potential resembles the moduli potential coming from supersymmetry breaking,

$$
V_{\text {susy }}(\phi)=m_{\text {susy }}^{4} v\left(\frac{\phi}{M_{P l}}\right),
$$

where $m_{\text {susy }}$ stands for the supersymmetry breaking scale. Potentials with such form were used in the inflationary context in refs. 24. In our context, eq. (6) implies that $m_{\text {susy }} \sim 10^{16} \mathrm{Gev}$. That is, the susy breaking scale $m_{\text {susy }}$ turns out to be at the GUT scale $m_{\text {susy }} \sim M_{G U T}$.

We see that the mass scale of the inflaton $m \sim$ $10^{13} \mathrm{GeV}$ can be related with $M_{G U T}$ by a see-saw style relation eq.(5).

As discussed in sec. 2 the inflaton describes a condensate in a GUT theory in which it may describe fermion-antifermion pairs. Current identifications in the literature of such condensate field with a given fundamental field in a SUSY or SUGRA model have so far no solid basis. Moreover, the number of supersymmetric models is so large that there is practically no way to predict which is the correct model [27.

In order to generate inflation in string theory, one needs first to generate a mass scale like $m \sim$ $10^{13} \mathrm{GeV}$ and $M_{G U T}$ related by eq. (5). Without the presence of the mass scales $m$ and $M_{G U T}$ [related through eq.(51)], there is no hope in string theory to get a correct inflationary cosmology describing the observed CMB fluctuations 28]. Such scale is not present in the string action, neither in the action of the effective background fields (dilaton, graviton, antisymmetric tensor) which are massless. Such scale should be gen- erated dynamically perhaps from the string vacuum(ua) but this is still an open problem far from being solved 28. Actually, the very same problem hinders the derivation of a GUT theory and the generation of the GUT scale from string theory.

Since no microscopic derivation of an inflationary model from a GUT is available so far, it would seem too ambitious at this stage to look for a microscopic derivation of inflation from string theory. The derivation of an inflationary cosmology reproducing the observed CMB fluctuations is at present too far away in string theory. However, an effective description of inflation in string theory (string matter plus massless backgrounds) could be at reach 28 .

\section{The inflaton as a quantum field: observ- able consequences through the CMB fluctuations}

We review now quantum phenomena during inflation which contribute to relevant observables in the CMB anisotropies and polarization. In particular, we focus on inflaton decay during inflation as a potential source of quantum phenomena contributing to deviations from scale invariance in the primordial power spectrum and/or to non-gaussian features. If the inflaton couples to other particles, then its quantum fluctuations which seed scalar density perturbations also couple to these other fields. Consequently, the decay of the amplitude of the quantum fluctuations of the inflaton may lead to a modification of the power spectrum of density perturbations. The same coupling that is responsible for the decay of the inflaton quantum fluctuations can be also the source of non-gaussian correlations.

Particle decay is a distinct feature of interacting quantum field theories and is necessarily an important part of the inflationary paradigm: the decay of the inflaton into lighter particles after inflation may yield to the radiation dominated stage.

In ref. 2] we introduced and implemented a systematic program to study the relaxational dynamics and particle decay in the case of a rapidly expanding inflationary stage. Whereby rapid expansion refers to the Hubble parameter during 
inflation being much larger than the mass of the particles. In the case of the inflaton, this is the situation of relevance for slow-roll inflation and a necessary (although not sufficient) condition for an almost scale invariant power spectrum of scalar fluctuations [1].

The Minkowski space-time computation of the decay rate is not suitable for a stage of rapid expansion (as quantified above): the rapid expansion of the Universe and the manifest lack of a global time-like Killing vector allow processes that would be forbidden by energy conservation in Minkowski space-time. As emphasized in 219, the lack of energy conservation in a rapidly expanding cosmology requires a different approach to study particle decay. The correct decay law follows from the relaxation in time of the expectation value of the field out of equilibrium. The relaxation of the non-equilibrium expectation value of the field is computed in ref. 2] using the dynamical renormalization group (DRG) which allows to extract the decay law directly from the real time equations of motion. The reliability and predictive power of the DRG has been tested for a wide range of physical situations including hot and dense plasmas in and out of equilibrium [20].

The goals of this work: We compute the particle decay of quantum fields minimally coupled to gravity with masses $M$ much smaller than the Hubble parameter, which is the relevant case for slow roll inflation 2]. This entails a much stronger infrared behavior than for massless particles conformally coupled to gravity 2 . The emergence of infrared divergences in quantum processes with gravitons during de Sitter inflation has been the focus of a thorough study 2122. As we will see below, similar strong infrared behavior enters in the decay of minimally coupled particles with masses $M$ much smaller than the Hubble parameter $H$. When $M<<H$ there is a small parameter $\Delta \sim M^{2} / H^{2}$ which regulates the infrared behavior in de Sitter inflation.

We began by studying the general case of a cubic interaction of scalar particles minimally coupled to gravity, allowing the decay of one field into two others during de Sitter inflation 2]. The masses of all particles are much smaller than the Hubble constant, which leads to a strong infrared behavior in the self-energy loops. We introduced an expansion in terms of the small parameter $\Delta$ which regulates the infrared and which in the case of de Sitter inflation is determined by the ratio of the mass squared of the particle in the loop to the the Hubble constant. Long-time divergences associated with secular terms in the solutions of the equations of motion are systematically resummed by implementing the DRG introduced in refs. 220 and lead to the decay law.

We then applied these general results to the case of quasi-de Sitter slow roll inflation [2]. We showed that in this case a similar small parameter $\Delta$ emerges which is a simple function of the slowroll parameters, and which regulates the infrared behavior even for massless particles (gravitons). We studied the decay of superhorizon fluctuations as well as of fluctuations with wavelengths deep inside the horizon. A rather striking aspect is that a particle can decay into itself precisely as a consequence of the lack of energy conservation in a rapidly expanding cosmology. We then focus on studying the decay of the inflaton quantum fluctuations into their own quanta, namely the self-decay of the inflaton fluctuations, discussing the potential implications on the power spectra of primordial perturbations and to non-gaussianity.

\section{Brief summary of results:}

- In the case of de Sitter inflation for particles with mass $M \ll H$, a small parameter $\Delta \sim M^{2} / H^{2}$ regulates the infrared. We introduce an expansion in this small parameter $\Delta$ akin to the $\varepsilon$ expansion in dimensionally regularized critical theories. We obtain the decay laws in a $\Delta$ expansion after implementing the DRG resummation.

- Minimally coupled particles decay faster than those conformally coupled to gravity due to the strong infrared behavior both for superhorizon modes as well as for modes with wavelengths well inside the Hubble radius.

- The decay of short wavelength modes, those inside the horizon during inflation, is en- 
hanced by soft collinear bremsstrahlung radiation of superhorizon quanta which becomes the dominant decay channel when the physical wave vector obeys,

$$
k_{p h}(\eta) \equiv \frac{k}{a(\eta)} \lesssim \frac{H}{\eta_{V}-\epsilon_{V}},
$$

where $\eta_{V}, \epsilon_{V}$ are the standard slow roll parameters.

- An expanding cosmology allows processes that are forbidden in Minkowski space-time by energy conservation 219]: in particular, for masses $\ll H$, kinematic thresholds are absent allowing a particle to decay into itself. Namely, the self-decay of quantum fluctuations is a feature of an interacting theory in a rapidly expanding cosmology. A self-coupling of the inflaton leads to the self-decay of its quantum fluctuations both for modes inside as well as outside the Hubble radius.

- The results obtained for de Sitter expansion directly apply to the self decay of the quantum fluctuations of the inflaton during slow roll (quasi de Sitter) expansion. In this case, $\Delta$ is a simple function of the slow roll parameters. For superhorizon modes we find that the amplitude of the inflaton quantum fluctuations relaxes as a power law $\eta^{\Gamma}$ in conformal time. To lowest order in slow roll, we find $\Gamma$ completely determined by slow roll parameters and the amplitude of the power spectrum of curvature perturbations $\triangle_{\mathcal{R}}^{2}$ :

$$
\Gamma=\frac{8 \xi_{V}^{2} \triangle_{\mathcal{R}}^{2}}{\left(\epsilon_{V}-\eta_{V}\right)^{2}}\left[1+\mathcal{O}\left(\epsilon_{V}, \eta_{V}\right)\right]
$$

where $\eta$ is conformal time and $\xi_{V}, \eta_{V}, \epsilon_{V}$ are the standard slow roll parameters. As a consequence, the growing mode which dominates at late time evolves as

$$
\frac{\eta^{\eta_{V}-\epsilon_{V}+\Gamma}}{\eta} \text {. }
$$

featuring a new scaling dimension $\Gamma$ slowing down the growth of the dominant mode.
The decay of the inflaton quantum fluctuations with wavelengths deep within the Hubble radius during slow roll inflation is enhanced by the infrared behavior associated with the collinear emission of ultrasoft quanta, namely bremsstrahlung radiation of superhorizon fluctuations. The decay hastens as the physical wavelength approaches the horizon because the phase space for the emission of superhorizon quanta opens up as the wavelength nears horizon crossing.

- We discuss the implications of these results for scalar and tensor perturbations, and establish a connection with previous calculations of non-gaussian correlations.

\section{Quantum Inflaton Decay}

We consider a general interacting scalar quantum field theory with cubic couplings in a spatially flat cosmological Friedmann-RobertsonWalker space time with scale factor $a(t)$. The cubic couplings are the lowest order non-linearities. Our study applies to two different scenarios, i) the inflaton $\phi$ coupled to another scalar field $\varphi$, ii) the inflaton field self-coupled via a trilinear coupling. We consider the fields to be minimally coupled to gravity.

In comoving coordinates the action for case i) is given by

$$
\begin{aligned}
& A=\int d^{3} x d t a^{3}(t)\left\{\frac{1}{2} \dot{\phi}^{2}-\frac{(\nabla \phi)^{2}}{2 a^{2}}-\frac{1}{2} M^{2} \phi^{2}+\right. \\
& +\frac{1}{2} \dot{\varphi}^{2}-\frac{(\nabla \varphi)^{2}}{2 a^{2}}-\frac{1}{2} m^{2} \varphi^{2}-g \phi \varphi^{2}+J(t) \phi+ \\
& \text { +higher nonlinear terms }\}
\end{aligned}
$$

and for the case ii),

$$
\begin{aligned}
& A=\int d^{3} x d t a^{3}(t)\left\{\frac{1}{2} \dot{\phi}^{2}-\frac{(\nabla \phi)^{2}}{2 a^{2}}-\frac{1}{2} M^{2} \phi^{2}+\right. \\
& \left.+\frac{g}{3} \phi^{3}+J(t) \phi+\text { higher nonlinear terms }\right\}
\end{aligned}
$$


The linear term in $\phi$ is a counterterm that will be used to cancel the tadpole diagram in the equations of motion. The higher nonlinear terms do not affect our results but they are necessary to stabilize the theory.

We computed in these two models the quantum decay of the inflaton from the self-energy corrections to the equations of motion to one-loop order [2].

We worked in conformal time $\eta$ with $d \eta=$ $d t / a(t)$ and introduce a conformal rescaling of the fields

$$
a(t) \phi(\vec{x}, t)=\chi(\vec{x}, \eta) ; \quad a(t) \varphi(\vec{x}, t)=\delta(\vec{x}, \eta) .
$$

The action Eq. (10) (after discarding surface terms that do not affect the equations of motion) reads:

$$
\begin{aligned}
& A[\chi, \delta]=\int d^{3} x d \eta\left\{\frac{\chi^{\prime 2}}{2}-\frac{(\nabla \chi)^{2}}{2}\right. \\
& -\frac{\mathcal{M}_{\chi}^{2}(\eta)}{2} \chi^{2}+\frac{\delta^{\prime 2}}{2}-\frac{(\nabla \delta)^{2}}{2}-\frac{\mathcal{M}_{\delta}^{2}(\eta)}{2} \delta^{2} \\
& \left.-g C(\eta) \chi \delta^{2}+C^{3}(\eta) J(\eta) \chi\right\}
\end{aligned}
$$

with primes denoting derivatives with respect to conformal time $\eta, C(\eta)=a(t(\eta))$ being the scale factor as a function of $\eta$ and

$$
\begin{aligned}
& \mathcal{M}_{\chi}^{2}(\eta)=M^{2} C^{2}(\eta)-\frac{C^{\prime \prime}(\eta)}{C(\eta)}, \\
& \mathcal{M}_{\delta}^{2}(\eta)=m^{2} C^{2}(\eta)-\frac{C^{\prime \prime}(\eta)}{C(\eta)} .
\end{aligned}
$$

For de Sitter space time, the scale factor is given by:

$$
a(t)=e^{H t}, C(\eta)=-\frac{1}{H \eta}, \eta=-\frac{e^{-H t}}{H},
$$

with $H$ the Hubble constant and where $\eta=-\frac{1}{H}$ corresponds to the initial time $t=0$.

The Heisenberg equations of motion for the Fourier field modes of wave vector $k$ in the free $(g=0)$ theory are given by

$$
\chi_{\vec{k}}^{\prime \prime}(\eta)+\left[k^{2}-\frac{1}{\eta^{2}}\left(\nu^{2}-\frac{1}{4}\right)\right] \chi_{\vec{k}}(\eta)=0,
$$

$$
\delta_{\vec{k}}^{\prime \prime}(\eta)+\left[k^{2}-\frac{1}{\eta^{2}}\left(\bar{\nu}^{2}-\frac{1}{4}\right)\right] \delta_{\vec{k}}(\eta)=0,
$$

where

$$
\nu^{2}=\frac{9}{4}-\frac{M^{2}}{H^{2}} \quad, \quad \bar{\nu}^{2}=\frac{9}{4}-\frac{m^{2}}{H^{2}} .
$$

The Heisenberg free field operators can be expanded in terms of the linearly independent solutions of the mode equation

$$
S_{\nu}^{\prime \prime}(k ; \eta)+\left[k^{2}-\frac{1}{\eta^{2}}\left(\nu^{2}-\frac{1}{4}\right)\right] S_{\nu}(k ; \eta)=0,
$$

for $\nu, \bar{\nu}$ respectively. We choose the usual BunchDavies initial conditions at $\eta \rightarrow-\infty$ for the mode functions, namely the usual plane waves for wavelengths deep inside the Hubble radius $|k \eta| \gg 1$. The mode functions $S_{\nu}(q, \eta)$ associated with the Bunch-Davies vacuum are given by

$$
S_{\nu}(k, \eta)=\frac{1}{2} i^{-\nu-\frac{1}{2}} \sqrt{\pi \eta} H_{\nu}^{(2)}(k \eta) .
$$

The modes with index $\nu$ are associated to the inflaton field $\phi$ while the modes with index $\bar{\nu}$ are associated to the lighter field $\varphi$. In the case of the de Sitter background eq.(14), $\nu$ and $\bar{\nu}$ are given by eqs. (15).

We showed in ref. 22 that during slow-roll inflation the background is quasi de Sitter and to lowest order in slow roll for the scalar perturbations is given by:

$$
C(\eta)=-\frac{1}{H \eta}\left(1+\epsilon_{V}\right)+\mathcal{O}\left(\epsilon_{V}^{2}\right)
$$

where $\epsilon_{V}$ is the usual the slow roll parameter (see for example 3 ). Therefore, for model ii) the parameters $\nu$ and $\Delta$ associated to the inflaton are given by 2

$$
\begin{aligned}
\nu & =\frac{3}{2}+\epsilon_{V}-\frac{M^{2}}{3 H^{2}}=\frac{3}{2}+\epsilon_{V}-\eta_{V}, \\
\Delta & \equiv \frac{3}{2}-\nu=\eta_{V}-\epsilon_{V},
\end{aligned}
$$

where $\eta_{V}$ is the usual the slow roll parameter (see for example [3]). Notice that the CMB anisotropy observations indicate that the slow roll parameters are of the order $10^{-2}$ and hence $\Delta$ is of the order $10^{-2}$ too . 
For gauge invariant scalar and tensor perturbations the infrared parameter $\Delta$ takes different forms. We find to first order in slow roll [2]:

$$
\begin{aligned}
& \Delta=-\eta_{V}-\epsilon_{V} \quad \text { scalar gauge invariant pert. } \\
& \Delta=-\epsilon_{V} \quad \text { tensor gauge invariant pert. }
\end{aligned}
$$

In ref. 2] we obtained the decay law for the quantum fluctuations of the inflaton field $\phi$ by using the equation of motion for the expectation value of $\phi$ and implementing the dynamical renormalization group (DRG). These equations follow from the non-equilibrium generating functional which involves forward and backward time evolution, typical of a density matrix. Unlike the S-matrix case (which is an in-out transition probability where only forward time evolution is required), the time evolution of an expectation value is an initial value problem which requires an in-in matrix element. Real time equations of motion obtained from the non-equilibrium generating functional are guaranteed to be retarded. In Fourier space the equations of motion for the expectation value of the field

$$
X_{\vec{k}}(\eta) \equiv\left\langle\chi_{\vec{k}}(\eta)\right\rangle
$$

are the integro-differential equation [2]

$$
\begin{aligned}
X_{\vec{k}}^{\prime \prime}(\eta) & +\left[k^{2}-\frac{\nu_{R}^{2}-\frac{1}{4}}{\eta^{2}}\right] X_{\vec{k}}(\eta)+g^{2} \frac{\delta M_{1}^{2}}{H^{2} \eta^{2}} X_{\vec{k}}(\eta) \\
& +\frac{2 g^{2}}{\eta H^{2}} \int_{\eta_{0}}^{\eta} \frac{d \eta^{\prime}}{\eta^{\prime}} \mathcal{K}_{\bar{\nu}}\left(k ; \eta, \eta^{\prime}\right) X_{\vec{k}}\left(\eta^{\prime}\right)=(2,0)
\end{aligned}
$$

where $\delta M_{1}^{2}$ stands for the UV mass renormalization and the kernel $\mathcal{K}_{\bar{\nu}}\left(k ; \eta, \eta^{\prime}\right)$ is given by the one-loop self-energy diagram. The kernel $\mathcal{K}_{\bar{\nu}}\left(k ; \eta, \eta^{\prime}\right)$ was computed in eq.(B14) of ref. 22]. It features a simple pole at $\bar{\Delta}=0$ :

$$
\begin{aligned}
\mathcal{K}_{\bar{\nu}}\left(k ; \eta, \eta^{\prime}\right)= & \frac{1}{4 \pi^{2} k^{3}\left(\eta \eta^{\prime}\right)^{2} \bar{\Delta}}\left\{k\left(\eta-\eta^{\prime}\right) \cos \left[k\left(\eta-\eta^{\prime}\right)\right]\right. \\
& \left.-\left(1+k^{2} \eta \eta^{\prime}\right) \sin \left[k\left(\eta-\eta^{\prime}\right)\right]\right\}+\mathcal{O}\left(\bar{\Delta}^{0}\right)
\end{aligned}
$$

The perturbative solution of Eq.(20) is obtained by writing

$$
X_{\vec{k}}(\eta)=X_{0, \vec{k}}(\eta)+g^{2} X_{1, \vec{k}}(\eta)+\mathcal{O}\left(g^{4}\right)
$$

The first order correction $X_{1, \vec{k}}(\eta)$ can be expressed by quadratures 2

$$
\begin{aligned}
X_{1, \vec{k}}(\eta) & =-\int_{\eta_{0}}^{0} d \eta^{\prime} \mathcal{G}_{\nu}\left(k ; \eta, \eta^{\prime}\right)\left[\frac{\delta M_{1}^{2}}{H^{2} \eta^{2}} X_{0, \vec{k}}\left(\eta^{\prime}\right)\right. \\
& \left.+\frac{2}{H^{2} \eta^{\prime}} \int_{\eta_{0}}^{\eta^{\prime}} \frac{d \eta^{\prime \prime}}{\eta^{\prime \prime}} \mathcal{K}_{\bar{\nu}}\left(k ; \eta^{\prime}, \eta^{\prime \prime}\right) X_{0, \vec{k}}\left(\eta^{\prime \prime}\right)\right]
\end{aligned}
$$

where $\mathcal{G}_{\nu}\left(k ; \eta, \eta^{\prime}\right)$ is the retarded Green's function

$\mathcal{G}_{\nu}\left(k ; \eta, \eta^{\prime}\right)=\theta\left(\eta-\eta^{\prime}\right) \frac{\pi}{2} \operatorname{Im}\left[H_{\nu}^{(1)}(k \eta) H_{\nu}^{(2)}\left(k \eta^{\prime}\right)\right]$,

and $H_{\nu}^{(1)}(z)$ and $H_{\nu}^{(2)}(z)$ stand for Hankel functions.

\section{Superhorizon Modes: $k=0$}

For superhorizon modes $(k=0)$ the general solution of the unperturbed mode equations (16) is given by

$$
X_{0, \overrightarrow{0}}(\eta)=A(-\eta)^{\beta_{+}}+B(-\eta)^{\beta_{-}} ; \beta_{ \pm}=\frac{1}{2} \pm \nu .
$$

We find for the first order correction $\mathcal{O}\left(g^{2}\right)$ from eqs.(21) and (22) 2]

$X_{\overrightarrow{0}}(\eta)=X_{0, \overrightarrow{0}}(\eta)\left[1+\Gamma \ln \frac{\eta}{\eta_{0}}+\right.$ non - secular terms $]$

with

$$
\begin{aligned}
& \Gamma=\frac{g^{2}}{16 \pi H^{2} \nu} \tan [\pi \nu]\left[1+\frac{4}{\frac{9}{4}-\nu^{2}}\right] \\
& =\frac{g^{2}}{16 \pi H^{2} \nu} \tan [\pi \nu]\left[1+\frac{4 H^{2}}{M^{2}}\right] .
\end{aligned}
$$

The term in $\ln \eta$ is a secular term since it grows unbounded with time implying a breakdown of perturbation theory. The dynamical renormalization group resummation 20 exponentiates the secular terms in Eq.(23) and leads to the improved solution [2],

$$
\begin{aligned}
& X_{\overrightarrow{0}}(\eta)=\left[\frac{\eta}{\eta_{0}}\right]^{\Gamma}\left\{A\left(\eta_{0}\right)(-\eta)^{\beta_{+}}\left[1+\mathcal{O}\left(g^{2}\right)\right]\right. \\
& \left.+B\left(\eta_{0}\right)(-\eta)^{\beta_{-}}\left[1+\mathcal{O}\left(g^{2}\right)\right]\right\}
\end{aligned}
$$

The first term inside the square bracket in Eq. (23) (namely the unit term) corresponds to the 
case in which the inflaton decays into massless particles conformally coupled to gravity 2 .

The calculation leading to eq.(23) is valid for $\bar{\nu} \rightarrow \frac{3}{2}$ (namely, $m \ll H$ ) and we keep $\nu$ as well as $M$ arbitrary. We can analytically continue the formula (23) to $H<M$ and then take the $m \ll H \ll M$ limit. In this limit $\Gamma$ becomes the decay rate of a particle with mass $M$ into massless particles in Minkowski space-time:

$$
\lim _{m \ll H \rightarrow 0} H \Gamma=\Gamma_{\text {Mink }}=g^{2} /(16 \pi M),
$$

as it must be.

\section{Conclusions and further questions}

We have reviewed here particle decay of fields minimally coupled to gravity in the case when the mass of the fields is $\ll H$ during inflation. Unlike the decay into massless fields conformally coupled to gravity, this case features a strong infrared behavior which leads to novel results.

We have implemented in ref. 2] the dynamical renormalization group resummation program introduced in ref. 20] combined with an expansion in a small parameter $\Delta$ which regulates the infrared.

In the case of exact de Sitter inflation, $\Delta$ is a constant equal to the ratio of the mass squared of the decay products to the Hubble constant squared, while in slow roll inflation $\Delta$ is a simple function of slow roll parameters. The expansion in $\Delta$ is akin to the $\varepsilon$ expansion in critical phenomena in dimensional regularization. The dynamical renormalization group provides a resummation of the long-time secular divergences which determine the decay law of quantum fluctuations.

The lack of energy conservation in an expanding cosmology leads to the lack of kinematic thresholds for particle decay. In particular, this possibility leads to the self-decay of quantum fluctuations whenever a self-interaction is present.

We have studied the decay of a particle for a cubic selfcoupled scalar field in de Sitter spacetime and applied the results to the self-decay of the inflaton quantum fluctuations during quasi de Sitter, slow roll inflation. We focused on extracting the decay law both for wavelengths well inside and well outside the Hubble radius. In both cases the strong infrared behavior enhances the decay.

The decay of fluctuations with wavelengths much smaller than the Hubble radius is enhanced by the collinear emission of ultrasoft quanta, this process is identified as bremsstrahlung radiation of superhorizon quanta. As the physical wavelength approaches the horizon, the phase space for this process opens up becoming the dominant decay channel for short wavelength modes in the region

$$
H \ll k_{p h}(\eta) \lesssim \frac{H}{\eta_{V}-\epsilon_{V}} .
$$

The decay of short wavelength modes hastens as the physical wavelength approaches the horizon as a consequence of the opening up of the phase space.

Superhorizon quantum fluctuations decay as a power law $\sim \eta^{\Gamma}$ in conformal time, where $\Gamma$ is determined by the following combination of the slow roll parameters and the amplitude of curvature perturbations

$$
\Gamma=\frac{32 \xi_{V}^{2} \triangle_{\mathcal{R}}^{2}}{\left(n_{s}-1+\frac{r}{4}\right)^{2}}\left[1+\mathcal{O}\left(\epsilon_{V}, \eta_{V}\right)\right]
$$

This decay law entails that the growing mode for superhorizon wavelengths evolves as $\eta^{\eta_{V}-\epsilon_{V}+\Gamma} / \eta$ hence $\Gamma$ provides a new scaling dimension slowing down the growing mode for late times $\eta \rightarrow 0^{-}$.

The recent WMAP data indicate that

3. $\times 10^{-8} \gtrsim \Gamma \gtrsim 3.6 \times 10^{-9}$.

This corresponds in cosmic time to a decay rate $10^{7} \mathrm{GeV} \gtrsim \Gamma_{\text {cosmic }} \equiv H \Gamma \gtrsim 10^{6} \mathrm{GeV}$.

Although these values may seem small, it must be noticed that the decay is a secular, namely cumulative effect.

Forthcoming observations of CMB anisotropies as well as large scale surveys with ever greater precision will provide a substantial body of high precision observational data which may hint at corrections to the generic and robust predictions of inflation. If such is the case these observations will pave the way for a better determination of inflationary scenarios. Studying the possible observational consequences of the quantum phenomena reviewed here will therefore prove a worthwhile endeavor. 


\section{REFERENCES}

1. E. W. Kolb and M. S. Turner, The Early Universe Addison Wesley, Redwood City, C.A. 1990. P. Coles and F. Lucchin, Cosmology, John Wiley, Chichester, 1995. A. R. Liddle and D. H. Lyth, Cosmological Inflation and Large Scale Structure, Cambridge University Press, 2000; Phys. Rep. 231, 1 (1993), and references therein.

2. D. Boyanovsky, H. J. de Vega, Phys. Rev. D70, 063508 (2004). D. Boyanovsky, H. J. de Vega, N. Sánchez, Phys. Rev. D 71, 023509 (2005).

3. D. Cirigliano, H. J. de Vega, N. Sánchez, astro-ph/0412634

4. V. A. Belinsky, L. P. Grishchuk, Ya. B. Zeldovich, I. M. Khalatnikov, Phys. Lett. B 155, 232, (1985), JETP 62, 195 (1985).

5. E. Komatsu et.al. (WMAP collaboration), Astro. Phys. Jour. Supp. 148, 119 (2003).

6. D. N. Spergel et.al. (WMAP collaboration), Astro. Phys. Jour. Supp. 148, 175 (2003).

7. A. Kogut et.al. (WMAP collaboration), Astro. Phys. Jour. Supp. 148, 161 (2003).

8. H. V. Peiris et.al. (WMAP collaboration), Astro. Phys. Jour. Supp. 148, 213 (2003).

9. D. N. Spergel and M. Zaldarriaga, Phys. Rev. Lett. 79, 2180 (1997); M. Zaldarriaga and D. D. Harari, Phys. Rev. D52, 3276 (1995).

10. T. J. Allen, B. Grinstein and M. B. Wise, Phys. Lett. B197, 66 (1987).

11. T. Falk, R. Rangarajan and M. Srednicki, Astrophys. J. 403, L1 (1993); Phys. Rev. D46, 4232 (1992).

12. A. Gangui, F. Lucchin, S. Matarrese and S.Mollerach, Astrophys. J. 430, 447 (1994).

13. J. Maldacena, JHEP 0305 , 013 (2003).

14. F. Bernardeau, T. Brunier and J.-P. Uzan, Phys.Rev. D69, 063520 (2004); F. Bernardeau and J.-P. Uzan, Phys.Rev. D67, 121301 (2003).

15. N. Bartolo, E. Komatsu, S. Matarrese and A. Riotto, astro-ph/0406398 (2004); V. Acquaviva, N. Bartolo,S. Matarrese and A. Riotto, Nucl. Phys. B667, 119 (2003).

16. D. Boyanovsky, H. J. de Vega, in the Proceedings of the VIIth. Chalonge School,
'Current Topics in Astrofundamental Physics', p. 37-57, edited by N. G. Sanchez, Kluwer publishers, Series C, vol. 562, (2001), astro-ph/0006446 D. Boyanovsky, D. Cormier, H. J. de Vega, R. Holman, S. P. Kumar, Phys. Rev. D 57, 2166 (1998). D. Boyanovsky, H. J. de Vega, R. Holman, Phys. Rev. D 49, 2769 (1994). D. Boyanovsky, F. J. Cao and H. J. de Vega, Nucl. Phys. B 632, 121 (2002), F. J. Cao, H. J. de Vega, N. G. Sanchez, Phys. Rev. D70, 083528 (2004).

17. D. Boyanovsky, R. Holman and S. Prem Kumar, Phys. Rev. D56, 1958 (1997).

18. S. Naidu and R. Holman, hep-th/0409013

19. R. P. Woodard, astro-ph/0310757.

20. D. Boyanovsky, H. J. de Vega, Ann. of Phys. 307, 335 (2003); D. Boyanovsky, H. J. de Vega, R. Holman and M. Simionato, Phys. Rev. D 60065003 (1999); D. Boyanovsky, H. J. de Vega, S.-Y. Wang, Phys.Rev. D67, 065022 (2003); D. Boyanovsky, H. J. de Vega, D.-S. Lee, S.-Y. Wang, H.-L. Yu, Phys.Rev. D65 , 045014 (2002); S.-Y. Wang, D. Boyanovsky, H. J. de Vega, D.-S. Lee, Phys.Rev. D62, 105026 (2000).

21. N. C. Tsamis and R. P. Woodard, Class. Quantum Grav. 11 2969, (1994); Ann. of Phys. (N.Y.) 238, 1 (1995).

22. A. D. Dolgov, M. B. Einhorn and V. I. Zakharov, Phys. Rev. D52, 717 (1995).

23. 'Supersymmetry Structure and Phenomena', N. Polonsky, Lect. Notes Phys. M68, 1-169 (2001), Springer-Verlag, Heidelberg. P. Langacker and N. Polonsky, Phys. Rev. D47, 4028 (1993). K. S. Babu, J. C. Pati, F. Wilczek, Nucl. Phys. B 56633 (2000).

24. F. C. Adams, J. R. Bond, K. Freese, J. A. Frieman, A. V. Olinto, Phys. Rev. 47, 426 (1993). G. Dvali, Q. Shafi and R. Schaefer, Phys. Rev. Lett. 73, 1886 (1994). V. N. Senaguz, Q. Shafi, hep-ph/0305189 and hep-ph/0412102 L. Randall, M. Soljacic, A. H. Guth, Nucl. Phys. B472, 377 (1996). K. Kadota, E. D. Stewart, hep-ph/0304127 and hep-ph/0311240

25. S. Weinberg, 'The Quantum Theory of Fields', vol. 3, Cambridge University Press, Cambridge, 2000. 
26. See for recent reviews, R. D. Peccei, hep-ph/0404277, G. Altarelli, F. Feruglio, hep-ph/0405048

27. P. Ramond, hep-ph/0411010

28. H. J. de Vega, N. Sánchez, Phys. Rev. D50, 7202 (1994), H. J. de Vega, N. Sánchez, 'Lectures on String Theory in Curved Spacetimes', at the Chalonge School, NATO ASI series 476, pp. 11-65, Kluwer Academic Publishers, Dordrecht, 1995, hep-th/9512074 M. P. Infante, N. Sánchez, Phys. Rev. D61, 083515 (2000). 